\title{
Severe malabsorption in autoimmune polyendocrinopathy-candidosis-ectodermal dystrophy syndrome successfully treated with immunosuppression
}

\author{
S Padeh, R Theodor, A Jonas, J H Passwell
}

\begin{abstract}
A 15 year old boy with autoimmune polyendocrinopathy-candidosis-ectodermal dystrophy syndrome suffered recurrent episodes of severe intractable diarrhoea, steatorrhoea, and hypocalcaemia. The only treatment modality, which controlled themalabsorptionsyndrome, wasimmunosuppression with intravenous high dose methylprednisolone and oral methotrexate maintenance therapy.

(Arch Dis Child 1997;76:532-534)
\end{abstract}

Keywords: autoimmune polyendocrinopathycandidosis-ectodermal-dystrophy syndrome; malabsorption; immunosuppression

Autoimmune polyendocrinopathy-candidosisectodermal dystrophy (APECED) is a rare autosomal recessive disease, characterised by variable endocrine deficiencies, chronic mucocutaneous candidiasis, and keratopathy. Prolonged periods of diarrhoea and intestinal malabsorption are uncommon features of the syndrome. ${ }^{1}$

We describe a patient with APECED who suffered recurrent episodes of severe intractable diarrhoea, steatorrhoea, and hypocalcaemia; this was controlled by immunosuppression with intravenous high dose methylprednisolone and oral methotrexate maintenance therapy.

\section{Case report}

A 15 year old boy first presented at the age of 2.5 years with a history of mucocutaneous candidiasis, alopecia totalis, muscle cramps, and tetany. The patient was the third son of Jewish consanguineous parents of Iranian ethnic origin. A sister died of Streptococcus pneumoniae septicaemia and red cell hypoplasia at the age of 8 years.

Hypoparathyroidism was confirmed by serum calcium $1.3 \mathrm{mmol} / 1$, serum phosphorus 2.7-3 mmol/l, tubular reabsorption of phosphorus $99 \%$, and low parathyroid hormone concentrations 2.5-10 pg/ml (normal 10-65 $\mathrm{pg} / \mathrm{ml}$ ), in the presence of hypocalcaemia. In addition subclinical hypoadrenalism was evident: low basal plasma cortisol $71.7 \mathrm{nmol} / 1$ (normal $>220 \mathrm{nmol} / \mathrm{l}$ ) with an abnormal response to adrenocorticotrophic hormone stimulation of $176.6 \mathrm{nmol} / 1$ (normal $>496$ nmol/1).

The diagnosis of APECED was made on the basis of these clinical symptoms, family history, and laboratory tests confirming hypoparathyroidism and hypoadrenalism.

The patient's disease course has been punctuated by recurrent episodes of tetany and episodes of diarrhoea with steatorrhoea; the coefficient of fat absorption (CFA) ranged between 15 and $35 \%$ (fig 1). These two symptoms were not always present at the same time, and serum calcium was maintained within the normal range when the CFA ranged between 65 and $85 \%$.

Repeated stool cultures for viruses and bacteria were all negative, as was staining for cryptosporidium and other parasites. No improvement in stool volume or steatorrhoea was noted after a prolonged course of ketoconazole $(6 \mathrm{mg} / \mathrm{kg} /$ day $)$ or metronidazole $(250 \mathrm{mg} /$ day $)$ treatment. During exacerbation, stool volumes ranged between 1-4 litres per day, did not respond to any elimination diet, but did to reduction in food or oral fluid intake. The stools showed large amounts of fat globules, fatty acid crystals, glucose and had a $\mathrm{pH}$ of 4-5. Stool electrolyte concentrations during exacerbation were sodium $35 \mathrm{mmol} / \mathrm{l}$, potassium 20 $\mathrm{mmol} / \mathrm{l}$, chloride $10 \mathrm{mmol} / \mathrm{l}$, thus excluding secretory diarrhoea. The d-xylose test was 1.1 $\mathrm{mmol} / \mathrm{l}$ at 60 minutes (normal $>1.7 \mathrm{mmol} / \mathrm{l}$ ). The lactulose breath test, barium meal and enema, endoscopy, and colonoscopy examinations gave normal results. An earlier jejunal biopsy specimen showed normal histology, but a later specimen showed slight villous shortening with mild subacute inflammation. Serum concentrations of intestinal hormones vasointestinal peptide, pancreatic polypeptide, gastrin, glucagon, neurosecretin, and somatostatin were normal.

Pancreatic function tests (Lundh and NBTPABA (bentiromide) test) were compatible with mild pancreatic insufficiency. However, his diarrhoea and steatorrhoea did not respond to 40 tablets/day of pancreatic extracts. Bile salt concentrations were within the normal range.

Immune studies showed a normal distribution of $\mathrm{T}$ lymphocyte subsets: CD11 $66 \%$, CD4 58\%, and CD8 20\%. Peripheral blood B lymphocytes CD20 8\% (IgG positive $2 \%$, IgM $5 \%, \kappa 2 \%$, and $\lambda 2 \%$ ). Raised serum immunoglobulin concentrations were detected: IgG $33.50 \mathrm{~g} / 1, \operatorname{IgA} 0.95 \mathrm{~g} / 1$ and $\operatorname{IgM} 2.50 \mathrm{~g} / 1$, while serum complement concentrations were normal. Candida skin test was negative, but lymphocyte proliferation to mitogens were within the normal range. Autoantibodies of all 


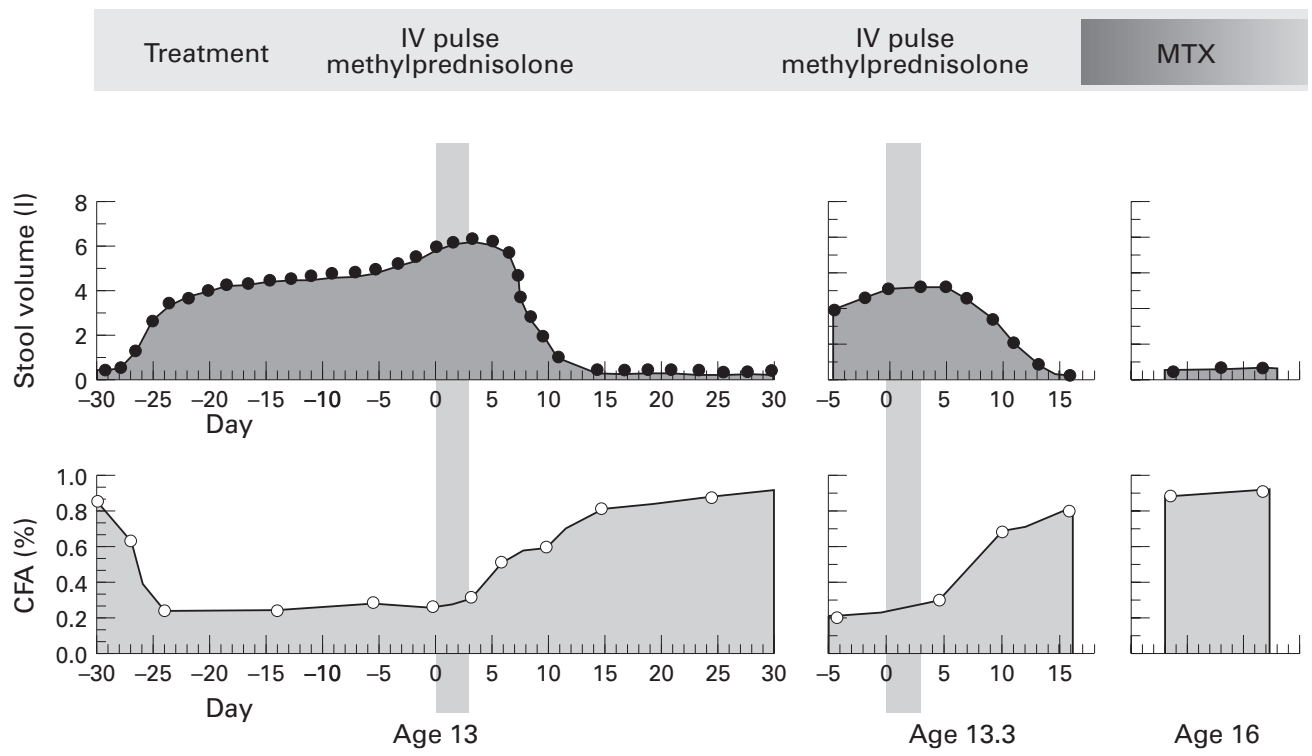

Figure 1 Response to intravenous (IV) pulse methylprednisolone during exacerbations of disease (CFA = coefficient of fat absorption; $M T X=$ methotrexate).

three immunoglobulin isotypes to the brush border of the normal gut enterocytes in the patient's serum were demonstrated by indirect immunofluorescence microscopy.

At the age of 6 a successful remission of disease was first achieved with high doses of intravenous steroid ( $2 \mathrm{mg} / \mathrm{kg} /$ day) and maintenance with oral prednisone, 1,25 dihydrocholecalciferol, and oral calcium supplements. Thereafter two exacerbations with tetany, severe malabsorption, and low serum calcium concentrations responded promptly to intravenous high dose methylprednisolone pulse therapy $(18 \mathrm{mg} / \mathrm{kg} /$ day) for three consecutive days. He has now been maintained free of gastrointestinal disease (CFA 93\%) on oral methotrexate $15 \mathrm{mg} / \mathrm{m}^{2}$ per week for the past three years (fig 1).

\section{Discussion}

The diagnosis of APECED in the reported patient was confirmed by the presence of hypoparathyroidism, chronic mucocutaneous moniliasis, alopecia totalis, and Addison's disease. Malabsorption and chronic diarrhoea were predominant symptoms in our patient, which precipitated hypocalcaemia and tetany. These recurrent episodes of diarrhoea and malabsorption did not respond to repeated and prolonged courses of broad spectrum antibiotics, metronidazole, or ketoconazole suggesting that an infectious aetiology was most unlikely. His severe diarrhoea did not improve during prolonged periods of normocalcaemia, nor with large amounts of pancreatic enzyme therapy. Thus although mild exocrine pancreatic insufficiency was demonstrated by laboratory tests, we considered that this did not play a major part in the pathogenesis of his disease.

APECED with autosomal recessive pattern of inheritance has been described in Israeli patients of Iranian ethnic origin. ${ }^{2}$ The fact that in our patient's family other members had clinical features of this syndrome, albeit without the gastrointestinal symptomatology, and his ethnic origin clearly indicate that he has this syndrome. A candidate gene for APECED, of a $5 \mathrm{~kb}$ cDNA coding a $3570 \mathrm{bp}$ protein, which has multiple putative transmembrane domains and partial homologies to transmembrane proteins including sodium channel proteins, has been mapped to human chromosome $21 \mathrm{q} 22.3$ by genetic linkage analysis. ${ }^{3}$ In addition, in other families an $\mathrm{X}$ linked inheritance pattern of disease with autoimmune enteropathy and various other immune mediated disease has been reported. ${ }^{4}$

The identification of mucosal antibodies and the repeated clinical remissions achieved with immunosuppressive treatment in the reported patient clearly support the idea that the intestinal disease is also part of the autoimmune syndrome. In fact the patient fulfils the four criteria of 'autoimmune enteropathy' first defined by Unsworth and Walker-Smith ${ }^{5}$ : (a) protracted diarrhoea and severe enteropathy; (b) no response to exclusion diet or total parental nutrition; (c) evidence for predisposition to autoimmune disease; and (d) no severe immune deficiency. Autoimmune enteropathy associated with hypothyroidism and diabetes mellitus has also been described. ${ }^{6}$ We speculate that in parallel to other autoimmune diseases, such as thyrotoxicosis or myasthenia gravis, specific antibodies may result in an as yet undetermined abnormal function of intestinal enterocytes that are responsible for the severe diarrhoea.

The clinical response to intravenous pulse steroids, although short lived, prompted long term treatment with a continuous immunosuppressive agent to control the diarrhoea. Methotrexate treatment was chosen because it is well tolerated in children with juvenile rheumatoid arthritis. Indeed, methotrexate in our patient controlled both diarrhoea and normal serum calcium concentrations were maintained with only a minimal requirement of vitamin D and calcium supplements. 
1 Ahonen P, Myllarniemi S, Sipila I, Perheentupa J. Clinical variation of autoimmune polyendocrinopathy-candidosis-
ectodermal dystrophy (APECED) in a series of 68 patients. ectodermal dystrophy (APECED)

N Engl f Med 1990;322:1829-36.
2 Zlotogora J, Shapiro MS. Polyglandular autoimmune Zlotogora J, Shapiro MS. Polyglandular autoimmune
syndrome type I among Iranian Jews. 7 Med Genet 1992;29: syndrome.

3 Yamakawa K, Mitchell S, Hubert R, et al. Isolation and characterization of a candidate gene for progressive myoclonus epilepsy on 21q22.3. Hum Mol Genet 1995;4:709-16.
4 Powell B, Buist N, Stenzel P. An X-linked syndrome of diarrhea, polyendocrinopathy, and fatal infection in infancy. F Pediatr 1982;100:731-7.

5 Unsworth D, Walker-Smith J. Autoimmunity in diarrheal disease. F Pediatr Gastroenterol Nutr 1985;4:375-81.

6 Savage O, Mirakian R, Wozniak ER, et al. Specific autoantibodies to gut epithelium in two infants with severe
protracted diarrhea. $\mathcal{F}$ Pediatr Gastroenterol Nutr 1985;4: protracted 